\title{
The Role of Multislice Computed Angiography of the Bronchial Arteries before Arterial Embolization in Patients with Hemoptysis
}

\author{
Despina Savvidou ${ }^{1 *}$, Katerina Malagari' ${ }^{2}$, Matina Kampanarou ${ }^{3}$, Fotios Laspas ${ }^{1}$, \\ Arkadios Rousakis ${ }^{1}$, Dimitrios Kelekis ${ }^{3}$, John Andreou' ${ }^{1}$ \\ ${ }^{1}$ CT-MRI Department, Hygeia Hospital, Athens, Greece \\ ${ }^{2}$ Research Unit of Radiology and Medical Imaging, Evgenidion Hospital, Athens, Greece \\ ${ }^{3}$ 2nd Department of Radiology, University of Athens, Athens, Greece \\ Email: * despinasavvidou@yahoo.com
}

Received 26 June 2014; revised 25 July 2014; accepted 25 August 2014

Copyright (C) 2014 by authors and Scientific Research Publishing Inc.

This work is licensed under the Creative Commons Attribution International License (CC BY). http://creativecommons.org/licenses/by/4.0/ (c) (i)

\section{Abstract}

Purpose: To evaluate the role of multislice computed angiography of the bronchial arteries and nonbronchial systemic arteries in patients with hemoptysis when performed before arterial embolization procedure. Materials and Methods: Twenty-eight patients with hemoptysis underwent multislice CT angiography of the bronchial arteries with dual-source $64 \times 2$ detector row scanner before embolization. The transverse CT images as well as the multiplanar reconstructions, the maximum intensity projections and the three-dimensional CT images were used for the depiction of bronchial arteries (the total number of the bronchial arteries, the abnormal bronchial arteries, their origin at the aorta and the diameter of the ostium). The presence of nonbronchial systemic arteries regarded as causing hemoptysis was also evaluated. Digital angiography and selective arteriograms of abnormal bronchial and nonbronchial systemic arteries were performed based on the findings of multislice computed tomography (MDCT). Results: Seventy-eight (40 right and 38 left) bronchial arteries were detected at computed angiography (CTA). Forty of the seventy-eight bronchial arteries that were detected at CTA, were considered abnormal. On selective angiography 38 of these bronchial arteries were regarded as causing hemoptysis. Two of these arteries could not be selectively catheterized and therefore could not be evaluated. All 38 bronchial arteries regarded as causing hemoptysis at selective angiography were detected prospectively at CTA as abnormal. Four bronchial arteries that were found to be responsible for hemoptysis had diameter $<2 \mathrm{~mm}$. Twelve nonbronchial systemic arteries were considered to be abnormal on CTA

\footnotetext{
"Corresponding author.
}

How to cite this paper: Savvidou, D., Malagari, K., Kampanarou, M., Laspas, F., Rousakis, A., Kelekis, D. and Andreou, J. (2014) The Role of Multislice Computed Angiography of the Bronchial Arteries before Arterial Embolization in Patients with Hemoptysis. Open Journal of Medical Imaging, 4, 133-141. http://dx.doi.org/10.4236/ojmi.2014.43019 
scans. Ten of these twelve nonbronchial systemic arteries were regarded on selective angiography as causing hemoptysis. Two of these arteries were found normal on angiography. All 10 nonbronchial arteries regarded as causing hemoptysis were detected at CTA scans. All bronchial and nonbronchial arteries causing hemoptysis were successfully embolized. Conclusion: MDCT angiography allows detailed identification of abnormal bronchial and nonbronchial systemic arteries using a variety of reformatted images, providing a precise road map for the interventional radiologist.

\section{Keywords}

\section{Hemoptysis, Bronchial Arteries, CTA, Embolization}

\section{Introduction}

Hemoptysis is an important symptom that needs careful investigation.

Bronchial arteries are the most common source of hemoptysis [1], despite the fact that they account for $1 \%$ of the arterial supply of the lungs. Nonbronchial systemic arteries may also be a source of hemoptysis in some instances. Bronchial artery embolization is an established nonsurgical treatment in the management of massive and recurrent hemoptysis [2]-[4]. Detection of the origins and the sources of the abnormal bronchial as well as nonbronchial arteries, before systemic therapeutic embolization, is very important information for the interventional radiologist.

After the advent of multislice computed tomography (MDCT), various retrospective studies [1]-[4] have shown the utility of multislice computed angiography (MDCTA).

MDCTA allows depiction of bronchial and nonbronchial systemic arteries using a combination of reformatted images in multiple planes [2]-[4].

Only one prospective study, in our knowledge, exist by Gypta et al. [5], showing the usefulness of MDCTA before arterial embolization.

The purpose of our study was to prospectively evaluate the role of MDCTA of the abnormal bronchial and nonbronchial arteries in patients with hemoptysis when used before arterial embolization procedures.

\section{Materials and Methods}

\subsection{Patients}

From October 2011 until September 2013, 28 patients (16 men and 12 women, age range 22 - 75 years) with hemoptysis underwent multislice CT angiography at our hospital as part of the pretherapeutic evaluation. Five patients had a history of embolization of bronchial arteries. Most patients were suffering from an underlying chronic disease that was considered to be responsible for hemoptysis: bronchiectasis in 12 patients (8 of them suffering from cystic fibrosis), chronic bronchitis and emphysema in 8 patients, tuberculosis in 3 patients, and secondary lung depositions in 1 patient (Table 1 ).

Digital angiography (DSA) was performed based on the findings of MDCT.

A comparison of the imaging studies was performed in these patients.

Table 1. Underlying disease in 28 patients with hemoptysis.

\begin{tabular}{cc}
\hline \multicolumn{1}{c}{ Bronchiectasis } & 12 pts (8/12 suffering from cystic fibrosis) \\
\hline Chronic bronchitis and emphysema & 8 patients \\
Tuberculosis & 3 patients patients \\
Secondary lung depositions & 4 patients \\
No underlying disease
\end{tabular}




\subsection{CT Technique and Interpretation}

Multislice CT angiography of the bronchial and nonbronchial systemic arteries was performed in 28 patients.

MDCTAs were performed with a $64 \times 2$ detector row helical scanner (Definition 64, Siemens). 80 - $100 \mathrm{ml}$ of contrast material and $40 \mathrm{ml}$ of saline was administered intravenously in all patients, with an injection rate of 5 $\mathrm{ml} / \mathrm{sec}$. A circular region of interest was placed at the ascending aorta with a threshold value of $100 \mathrm{HU}$ and scans began with a 6 sec delay. The scan range was from the lung apices to the level of the upper pole of the kidneys.

The lung parenchyma was studied in detail with $5 \mathrm{~mm}$ transverse scans in order to depict underlying disease and also localize the site of bleeding by identifying ground glass opacities or consolidations, findings that represent intralveolar hemorrhage.

Possible underlying causes of hemoptysis that can be identified at lung window settings include bronchiectasis, bronchogenic carcinoma, acute and chronic lung infections (tuberculosis and aspergillosis) [2] [6].

The origins of the bronchial arteries were depicted on axial $1 \mathrm{~mm}$ thick CT scans viewed at mediastinal window.

The diameter of the ostium of the bronchial arteries was measured on transverse images and multiplanar reconstruction images (MPR) parallel to the axis of their origin.

MPR images were obtained at various angles in order to evaluate the mediastinal course and the traceability of the bronchial arteries to the hilum.

3D images were acquired using volume-rendering (VRT) and maximum-intensity-projection (MIP) techniques to display the arteries as a whole on a single image.

Enhancing structures (dots or lines) in the posterior mediastinum that were connected to the aorta were considered as bronchial arteries [3] [4].

Bronchial arteries originating from the descending aorta between the levels of the T5 and T6 vertebrae are called orthotopic [3] [6].

When the bronchial arteries originate outside the T5 - T6 vertebral levels of the thoracic aorta or from any aortic collateral vessel (such as brachiocephalic artery, subclavian artery, internal mammary artery) are considered to be ectopic [6].

The total number of the bronchial arteries, the level and the side of their origin as well as their diameter were evaluated.

Bronchial arteries with a diameter $>2 \mathrm{~mm}$ at their origin and/or detectable to the hilum were considered on CTA as abnormal.

Nonbronchial systemic arteries (NBSAs) were also depicted. Arteries that entered the lung parenchyma through the adherent pleura or through the inferior pulmonary ligament and their course were not parallel to the bronchi were considered as nonbronchial arteries. NBSAs usually involved in hemoptysis are the intercostals arteries, the internal mammary arteries and other collaterals from the subclavian arteries or the inferior phrenic arteries [6].

When such systemic arteries were demonstrated enlarged within the extrapleural fat in association with pleural thickening (>3 mm), they were regarded as causing hemoptysis.

\subsection{Angiographic Procedure}

All patients underwent conventional angiography within 10 days after CT bronchial angiography. Of the 28 patients who underwent both CT and conventional angiography, 26 underwent bronchial artery embolization.

Angiographic procedures were performed based on the CT findings.

In all patients, conventional angiography was performed with Seldinger technique, with a digital substraction, using a transfemoral approach. A preliminary thoracic aortogram was performed and was followed by selective arteriogram of abnormal bronchial as well as nonbronchial arteries.

The angiographic criteria for arteries considered responsible for hemoptysis were: the diameter and the morphology of the vessels (enlarged and tortuous), as well as the presence of extravasation of contrast medium, areas of hypervascularity and neovascularity or bronchial artery aneurysm.

Embolic microspheres with diameter $>300 \mu \mathrm{m}$ were used for embolization of bronchial arteries that considered responsible for bleeding.

Both bronchial arteries and nonbronchial systemic arteries causing hemoptysis were recorded for each patient. 
The results of angiography were compared to the findings of MDCT.

\section{Results}

\subsection{CTA Findings}

Seventy-eight bronchial arteries were detected at CT angiography in 28 patients (40 right and 38 left).

The site of the origin of the majority (67/78) of the bronchial arteries was orthotopic.

Right bronchial arteries were considered orthotopic when they originated from an intercostobronchial trunk or from the descending aorta as a single artery at levels T5 - T6 (Figure 1) and left bronchial arteries when arose from the descending aorta as a single artery at levels T5 - T6.

The rest bronchial arteries originating from the thoracic aorta outside the T5 - T6 vertebral levels or from the aortic branches were considered as ectopic.

Four right bronchial arteries arose from the aortic arch.

Three left bronchial arteries arose from the aortic arch and four left bronchial arteries arose from the descending aorta, outside the T5 - T6 vertebral levels.

The diameter of each bronchial artery ranged from 1.2 to $5 \mathrm{~mm}$.

Forty of these bronchial arteries were considered as abnormal (25 right and 15 left bronchial arteries). The diameter of the ostium of these bronchial arteries ranged from $1.6-5 \mathrm{~mm}$.

Four bronchial arteries that were considered as abnormal on CTA, had diameter $<2 \mathrm{~mm}(1.6-1.9 \mathrm{~mm})$ at their origin but they were detectable to the hilum (Figure 2).

Five bronchial arteries causing hemoptysis had aberrant origin. Two right bronchial arteries arose from the aortic arch. Two left bronchial arteries arose from the descending aorta, outside the T5 - T6 vertebral levels (Figure 3) and one left bronchial artery from the aortic arch.

Twelve non-bronchial systemic arteries regarded as causing hemoptysis were detected on CT scans (10 intercostal arteries, one from left subclavian artery and one right internal mammary artery) in five patients. Three of these five patients had recurrent hemoptysis after bronchial artery embolization in the past.

\subsection{Angiographic Findings}

Thirty-eight bronchial arteries and 10 nonbronchial systemic arteries (8 intercostal arteries (Figure 4), one from left subclavian artery and one right internal mammary artery), were found to be responsible for hemoptysis.

All these bronchial and nonbronchial arteries were prospectively identified as abnormal on MDCT.

Two from forty bronchial arteries that were found to be abnormal on CTA could not be selectively catheterized and therefore could not be evaluated.

Two nonbronchial arteries that were found abnormal on MDCT were not regarded as causing hemoptysis at angiography.

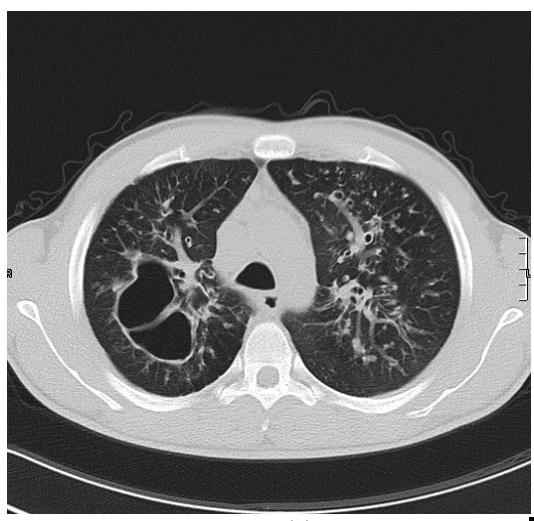

(a)

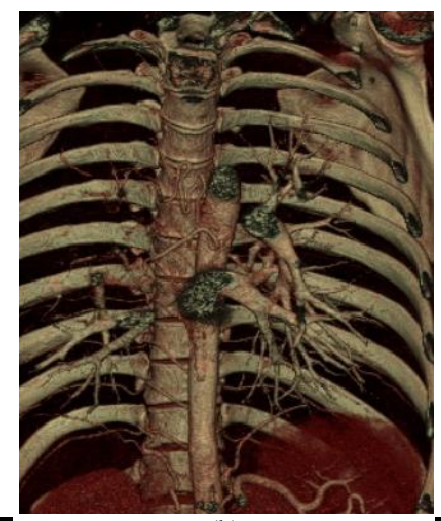

(b)

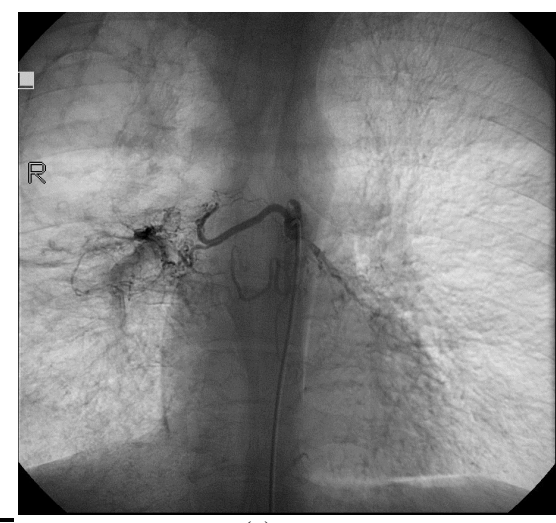

(c)

Figure 1. (a) Transverse $5 \mathrm{~mm}$ thick slice image (lung window) shows cystic bronchiectasis in both upper lung lobes. (b) VRT image show enlarged right bronchial arteries arising from the right anterior and lateral aspect of the descending aorta at the level of T6. (c) Selective angiogram of right enlarged and tortuous bronchial arteries with hypervascularity of the right upper lobe. 


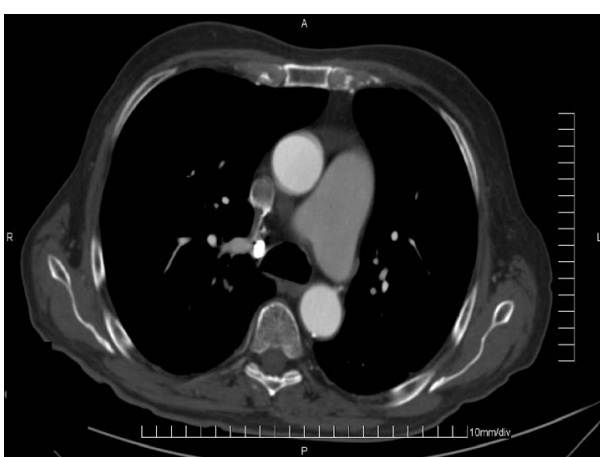

(a)

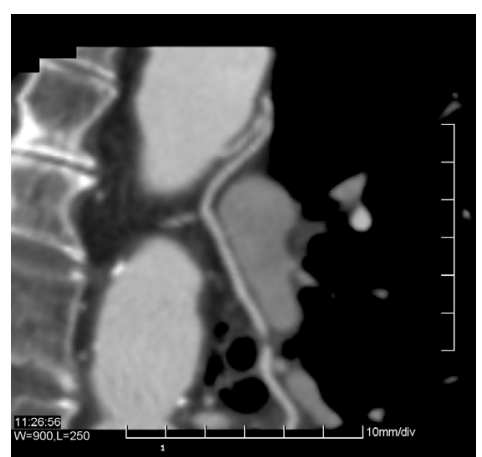

(b)

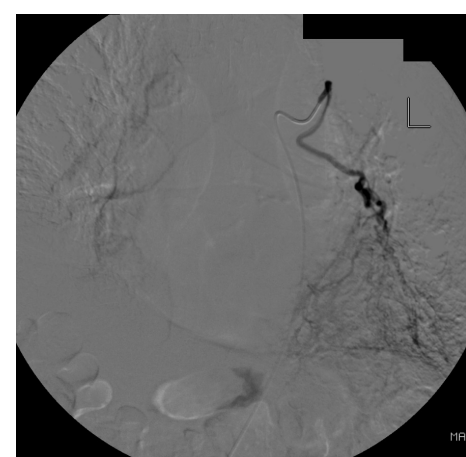

(c)

Figure 2. Images in a 50-year-old man with bronchiectasis of the upper lung lobes. (a) axial 1 mm thick slice CT image (mediastinal window) depicts the origin of a left bronchial artery arising from the descending aorta at the level of T6 vertebral, with diameter $1.8 \mathrm{~mm}$ at its origin. (b) MIP CT image shows left bronchial artery detectable to the hilum. (c) Selective bronchial angiography of the left bronchial artery with hypervascularity and microaneurysms.

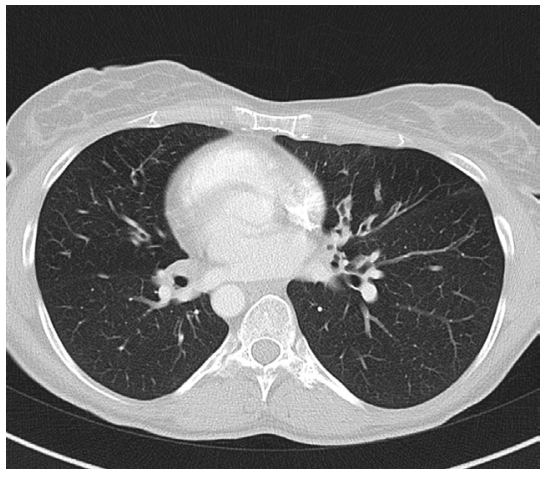

(a)

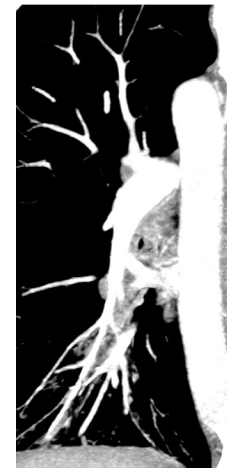

(b)

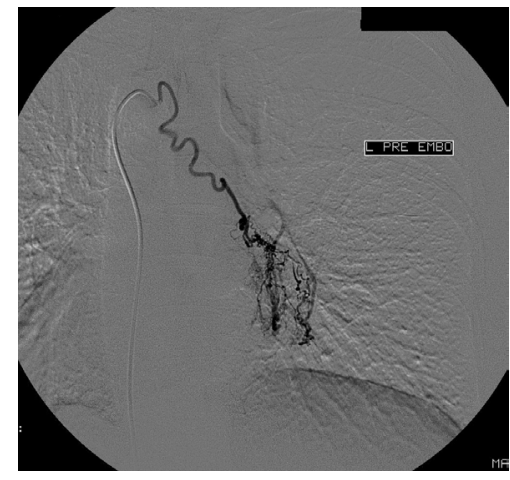

(c)

Figure 3. (a) Axial $5 \mathrm{~mm}$ thick slice CT image in lung window shows cystic bronchiectasis mainly in the left upper lobe. (b) MIP CT image in coronal plane depicts left enlarged ectopic bronchial artery originating from the descending aorta at the level of the T4 vertebral body. This bronchial artery is tortuous and traceable to the hilum. There is also right aortic arch. (c) Selective left bronchial angiogram shows left enlarged bronchial artery with hypervascularity of the left upper lobe.

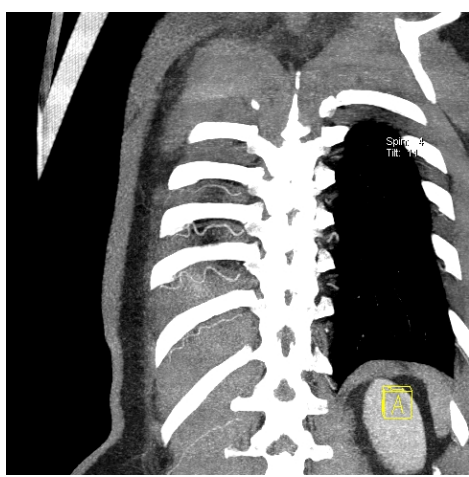

(a)

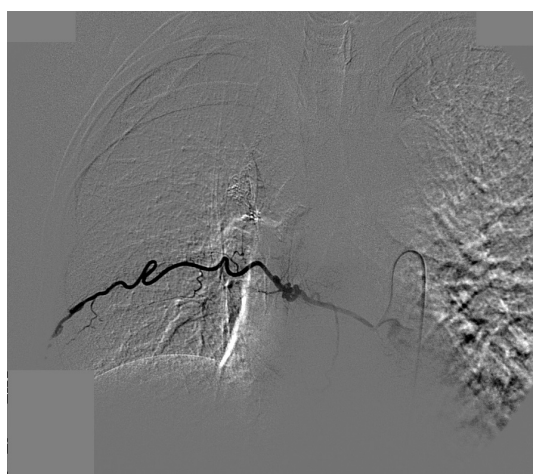

(b)

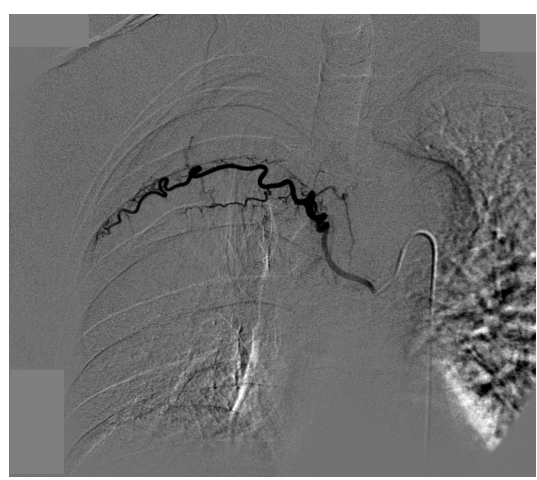

(c)

Figure 4. (a) MIP CT image in coronal plane shows multiple hypertrophied tortuous right intercostals arteries (b, c) Selective arteriograms showing two hypertrophied tortuous right intercostals arteries.

All bronchial and nonbronchial arteries that were considered responsible for hemoptysis were embolized.

Two abnormal bronchial arteries (one right bronchial artery from the aortic arch and one left orthotopic bronchial artery) as well as two abnormal nonbronchial arteries (one from left subclavian artery and the right internal mammary artery (Figure 5)) were not identified on flush aortogram. 
These 4 abnormal arteries were easily catheterized with the help of finding of CTA.

Table 2 and Table 3 compare the results of MDCTA and DSA in the depiction of bronchial and non-bronchial arteries considered as abnormal in 28 patients who underwent embolization.

\section{Discussion}

Bronchial arterial embolization is a proven effective, minimally invasive technique in the management of massive and recurrent hemoptysis. Hemoptysis can be a life-threatening respiratory emergency and needs urgent investigation.

Patients with hemoptysis are evaluated initially by a chest radiography, bronchoscopy and computed tomography in order to identify the site of bleeding and the underlying cause [6].

CT angiography is a very useful method in identifying bronchial and nonbronchial arteries using reformatted images in multiple projections [3] [4] [7] [8].

This information is very useful to the interventional radiologist for planning arterial embolization [6]-[9].

Bronchial arteries are small vessels arising directly from the descending aorta. They supply nutrient branches

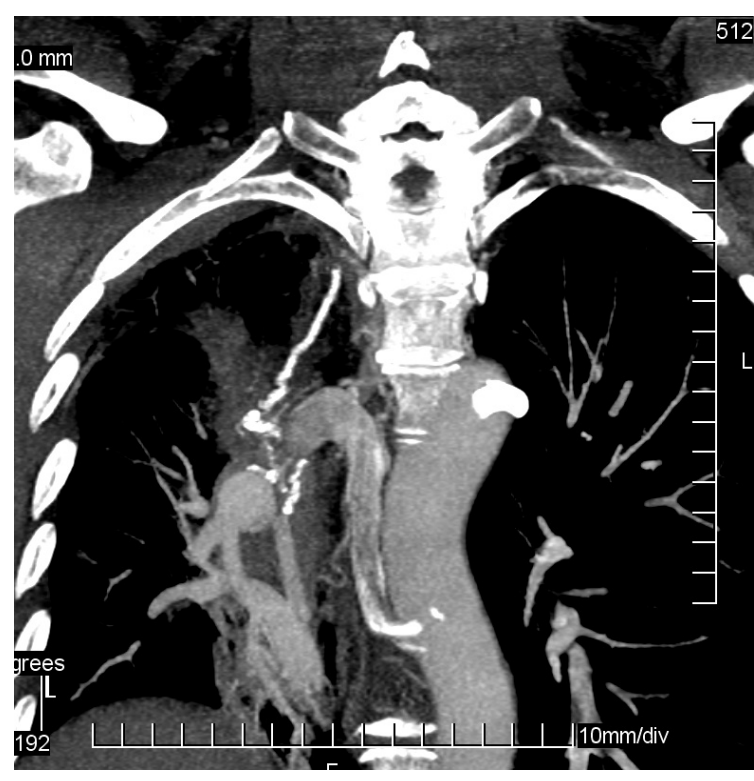

(a)

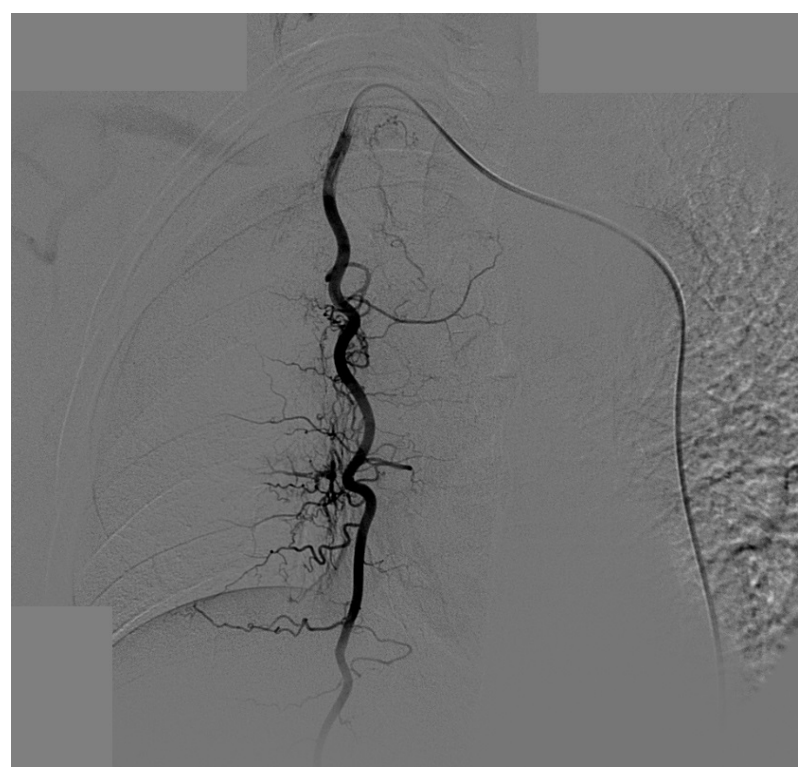

(b)

Figure 5. Images (a) MIP CT image shows tortuous right internal mammary artery. (b) Selective angiography depicts tortuous right internal mammary artery with hypervascularity and parenchymal staining of the right lung.

Table 2. Depiction of bronchial arteries (considered as abnormal) in 28 patients who underwent bronchial artery embolization.

CT Angiography

40 bronchial arteries were considered as abnormal
Conventional Angiography

38 bronchial arteries were found to be responsible for hemoptysis (all these were prospectively identified as abnormal in MDCTA)

2 bronchial arteries that considered as abnormal in MDCTA could not be selectively catheterized and therefore could not be evaluated

Table 3. Depiction of non-bronchial arteries in 28 patients who underwent bronchial artery embolization.

\begin{tabular}{cl}
\hline CT Angiography & \multicolumn{1}{c}{ Conventional Angiography } \\
\hline 12 non-bronchial arteries were considered as abnormal & $\begin{array}{l}10 \text { non-bronchial arteries were found to be responsible for hemoptysis } \\
\text { (all these were prospectively identified as abnormal in MDCTA) } \\
2 \text { non-bronchial arteries that were found abnormal in MDCTA were } \\
\text { not regarded as causing hemoptysis at angiography }\end{array}$ \\
\hline
\end{tabular}


to the airways of the lung, the esophagus, and the lymph nodes [2] [6].

Anatomy of the bronchial arteries regarding their origins and courses varies substantially. Most commonly, they arise from the descending aorta at the levels of T5 - T6 vertebral bodies and are called orthotopic bronchial arteries. Cauldwell et al. [10] described four main types of bronchial artery anatomy. Type 1 (40.6\%) has one right bronchial artery from right intercostobronchial trunk and two left bronchial arteries. Type 2 (21.3\%) has one right bronchial artery from an intercostobronchial trunk and one left. Type 3 (20.6\%) has two right bronchial arteries (one from an intercostobronchial trunk) and two left. Type 4 (9.7\%) has two right bronchial arteries (one from an intercostobronchial trunk) and one left. The most constant vessel is the right intercostobronchial trunk (88.7\%).

When bronchial arteries originate from the thoracic aorta outside the T5 - T6 vertebral levels or from the aortic branches, they are called anomalous or ectopic [6] [8].

Orthotopic, as well as, ectopic bronchial vessels enter the lung parenchyma through the hilum and course parallel to the main bronchi and their branches [2] [5] [8].

Bronchial arteries are the most common source of bleeding in hemoptysis. In these cases, in most patients, they are enlarged and tortuous.

They are identified, on CT images, as dots or lines of increased attenuation in the posterior mediastinum, around the central airways.

Enlarged bronchial arteries are most commonly found on CTA images, in the retrotracheal and retroesophageal areas, as well as the posterior wall of the main bronchus and the aortopulmonary window [4] [6].

Bronchial arteries that are larger than $2 \mathrm{~mm}$ in diameter on CT scans are considered to be abnormal and are candidates for embolotherapy, although bronchial bleeding may occur from normal-sized bronchial arteries [2][4] [11].

Yoon et al. [4] reported in a study comparing retrospectively MDCT with conventional angiography in patients with hemoptysis, that MDCT identified all bronchial arteries that were regarded at angiography as causing hemoptysis. The diameter of bronchial arteries which were abnormal on angiography, ranged from $1.3-4.7 \mathrm{~mm}$. Also 8 bronchial arteries causing hemoptysis were $<2 \mathrm{~mm}$.

In our study, all bronchial arteries (38) that were found to be responsible for hemoptysis on DSA were prospectively identified as abnormal on CTA.

In our study, 4 from 38 bronchial arteries that were regarded as causing hemoptysis at selective angiography, had a diameter smaller than $2 \mathrm{~mm}(1.6-1.8 \mathrm{~mm})$ at CTA but they were detectable to the hilum.

However, in a recent study Gupta et al. [5] used a cutoff value of $2 \mathrm{~mm}$ on MDCT to identify the abnormal bronchial arteries and prospectively detected with MDCT all abnormal bronchial arteries that were found responsible for hemoptysis on DSA.

A percentage of $86 \%$ of the bronchial arteries that were detected at CTA (67/78) were found to be orthotopic, originating from the descending aorta at the T5 - T6 level. These results are in agreement with previous reports [3] [10] [12], that emphasized that the majority ( $>70 \%)$ of the bronchial arteries arise from the descending aorta. As expected, the majority of the orthotopic right bronchial arteries (32 of 40) were originated from a right intercostobronchial trunk. All orthotopic left bronchial arteries were found originating from the descending aorta.

The rest (11/78) bronchial arteries observed at CTA had an ectopic origin.

The most frequent sites of origin of the ectopic bronchial arteries in our study, was the concavity of the aortic arch $(7 / 11,64 \%)$ and the descending aorta $(4 / 11,36 \%)$.

The most frequent sites of origin in the study of Hartman et al. [13] were the concavity of the aortic arch (74\%), the subclavian artery (10.5\%) and the descending aorta (8.5\%).

Our results are in agreement with Remy-Jardin et al. [3] that reported that the most frequent origin of ectopic bronchial arteries was the concavity of the aortic arch.

Five bronchial arteries that were considered as abnormal on CTA had aberrant origin. One right bronchial artery (originating from the aortic arch) of these five arteries was not identified on the preliminary flush thoracic decending aortogram. It was selectively catheterized based on the findings of CTA.

We confirmed that, three-dimensional images are more accurate than transverse CT scans for detecting bronchial arteries with ectopic origins, as Remy-Jardin et al. [3] also reported. This is very helpful for the interventional radiologists to perform direct catheterization of the ectopic vessels and successful embolization.

Nonbronchial systemic arteries may be the source of hemoptysis, especially in patients with recurrent hemoptysis [1]-[6]. These vessels are differentiated from the bronchial arteries by their courses. Nonbronchial ar- 
teries enter the lung parenchyma through the adherent pleura or the inferior pulmonary ligament and their course is not parallel to the bronchi. They can arise from the subclavian, axillary and internal mammary arteries, as well as infradiaphragmatic branches such as the inferior phrenic and left gastric arteries, and the celiac axis [2]-[5] [7] [8]. Enlarged and tortuous enhancing arteries detected within extrapleural fat in association with pleural thickening (3 mm) are regarded as nonbronchial systemic arteries causing hemoptysis [3] [4] [7] [14].

In our study, all nonbronchial systemic arteries regarded as causing hemoptysis on angiography were also detected prospectively as abnormal on CTA.

Our results are in agreement with the findings of a recent study by Gupta et al. [5], reporting that all nonbronchial arteries responsible for hemoptysis on DSA, were prospectively identified as abnormal on CTA.

CT angiographic findings enabled direct selective catheterization and embolization of abnormal nonbronchial systemic arteries.

A limitation of our study is that patients with significant hemodynamic compromise were not offered MDCTA before bronchial embolization in order to save time.

\section{Conclusion}

In conclusion, MDCT angiography allows rapid and detailed identification of abnormal bronchial and nonbronchial systemic arteries using a variety of reformatted images, providing a precise road map which can be used to guide therapeutic arterial embolization procedures. This information may be helpful for the interventional radiologist in order to avoid thoracic aortography and attempt direct catheterization of the arteries to be occluded, resulting in reducing the examination time in the angiography suite, and minimizing contrast load and radiation dose. So, CTA is recommended before conventional angiography and arterial embolization procedures.

\section{References}

[1] Khalil, A., Fartoukh, M., Tassart, M., Parrot, A., Marsault, C. and Carette, M.F. (2007) Role of MDCT in Identification of the Bleeding Site and the Vessels Causing Hemoptysis. AJR, 188, 117-125. http://dx.doi.org/10.2214/AJR.05.1578

[2] Chung, M.J., Lee, J.H., Lee, K.S., Yoon, Y.C., Kwon, O.J. and Kim, T.S. (2006) Bronchial and Nonbronchial Systemic Arteries in Patients with Hemoptysis: Depiction on MDCT Angiography. AJR, 186, 649-655. http://dx.doi.org/10.2214/AJR.04.1961

[3] Remy-Jardin, M., Bouaziz, N., Dumont, P., Brillet, P.Y. Bruzzi, J. and Remy, J. (2004) Bronchial and Nonbronchial Systemic Arteries at Multidetector Row CT Angiography: Comparison with Conventional Angiography. Radiology, 233, 741-749. http://dx.doi.org/10.1148/radiol.2333040031

[4] Yoon, Y.C., Lee, K.S., Jeong, Y.J., Shin, S.W., Chung, M.J. and Kwon, O.J. (2005) Hemoptysis: Bronchial and Nonbronchial Systemic Arteries at 16-Detector Row CT. Radiology, 234, 292-298. http://dx.doi.org/10.1148/radiol.2341032079

[5] Gupta, M., Srivastava, D.N., Seith, A., Sharma, S., Thulkar, S. and Gupta, R. (2013) Clinical Impact of Multidetector Row Computed Tomography before Bronchial Artery Embolization in Patients With Hemoptysis: A Prospective Study. Canadian Association of Radiologists Journal, 64, 61-73. http://dx.doi.org/10.1016/j.carj.2011.08.002

[6] Bruzzi, J.F., Remy-Jardin, M., Delhaye, D., Teisseire, A., Khalil, C. and Remy, J. (2006) Multi-Detector Row CT of Hemoptysis. Radiographics, 26, 3-22. http://dx.doi.org/10.1148/rg.261045726

[7] Ponnuswamy, I., Sankaravadivelu, S.T., Maduraimuthu, P., Natarajan, K., Sathyanathan, B.P. and Sadras, S. (2012) 64-Detector Row CT Evaluation of Bronchial and Non-Bronchial Systemic Arteries in Life-Threatening Haemoptysis. BJR, 85, 666-672. http://dx.doi.org/10.1259/bjr/24730002

[8] Yildiz, A.E., Ariyurek, O.M., Akpinar, E., Peynircioglu, B. and Cil, B.E. (2011) Multidetector CT of Bronchial and Non-Bronchial Systemic Arteries. Diagnostic Interventional Radiology, 17, 10-17.

[9] Noe, G.D., Jaffe, S.M. and Molan M.P. (2011) CT and CT Angiography in Massive Haemoptysis with Emphasis on Pre-Embolization Assessment. Clinical Radiology, 66, 869-875. http://dx.doi.org/10.1016/j.crad.2011.03.001

[10] Cauldwell, E.W., Siekert, R.G., Lininger, R.E. and Anson, B.J. (1948) The Bronchial Arteries: An Anatomic Study in 150 Human Cadavers. Surgery, Gynecology and Obstetrics, 86, 395-412.

[11] Mori, H., Ohno, Y., Tsuge, Y., Kawasaki, M., Ito, F., Endo, J., Funaguchi, N., La, B. L.B., Kanematsu, M. and Minatoguchi, S. (2010) Use of Multidetector Row CT to Evaluate the Need for Bronchial Arterial Embolization in Hemoptysis Patients. Respiration, 80, 24-31. http://dx.doi.org/10.1159/000253882

[12] Botenga A.S. (1970) Selective Bronchial and Intercostals Arteriography. Stenfert Kroese, Leiden, 53-65. http://dx.doi.org/10.1007/978-94-010-3156-1 
[13] Hartmann, I.J.C., Remy-Jardin, M., Menchini, L., Teisseire, A., Khalil, C. and Remy, J. (2007) Ectopic Origin of Bronchial Arteries: Assessment with Multidetector Helical CT Angiography. European Radiology, 17, 1943-1953. http://dx.doi.org/10.1007/s00330-006-0576-8

[14] Yoon, W., Kim, Y.H., Kim, J.K., Kim, Y.C., Park, J.G. and Kang, H.K. (2003) Massive Hemoptysis: Prediction of Nonbronchial Systemic Arterial Supply with Chest CT. Radiology, 227, 232-238. http://dx.doi.org/10.1148/radiol.2271020324 
Scientific Research Publishing (SCIRP) is one of the largest Open Access journal publishers. It is currently publishing more than 200 open access, online, peer-reviewed journals covering a wide range of academic disciplines. SCIRP serves the worldwide academic communities and contributes to the progress and application of science with its publication.

Other selected journals from SCIRP are listed as below. Submit your manuscript to us via either submit@scirp.org or Online Submission Portal.
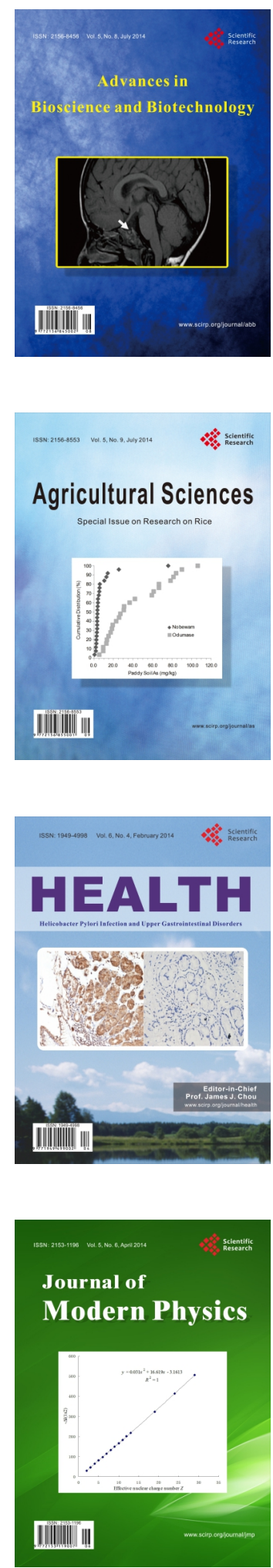
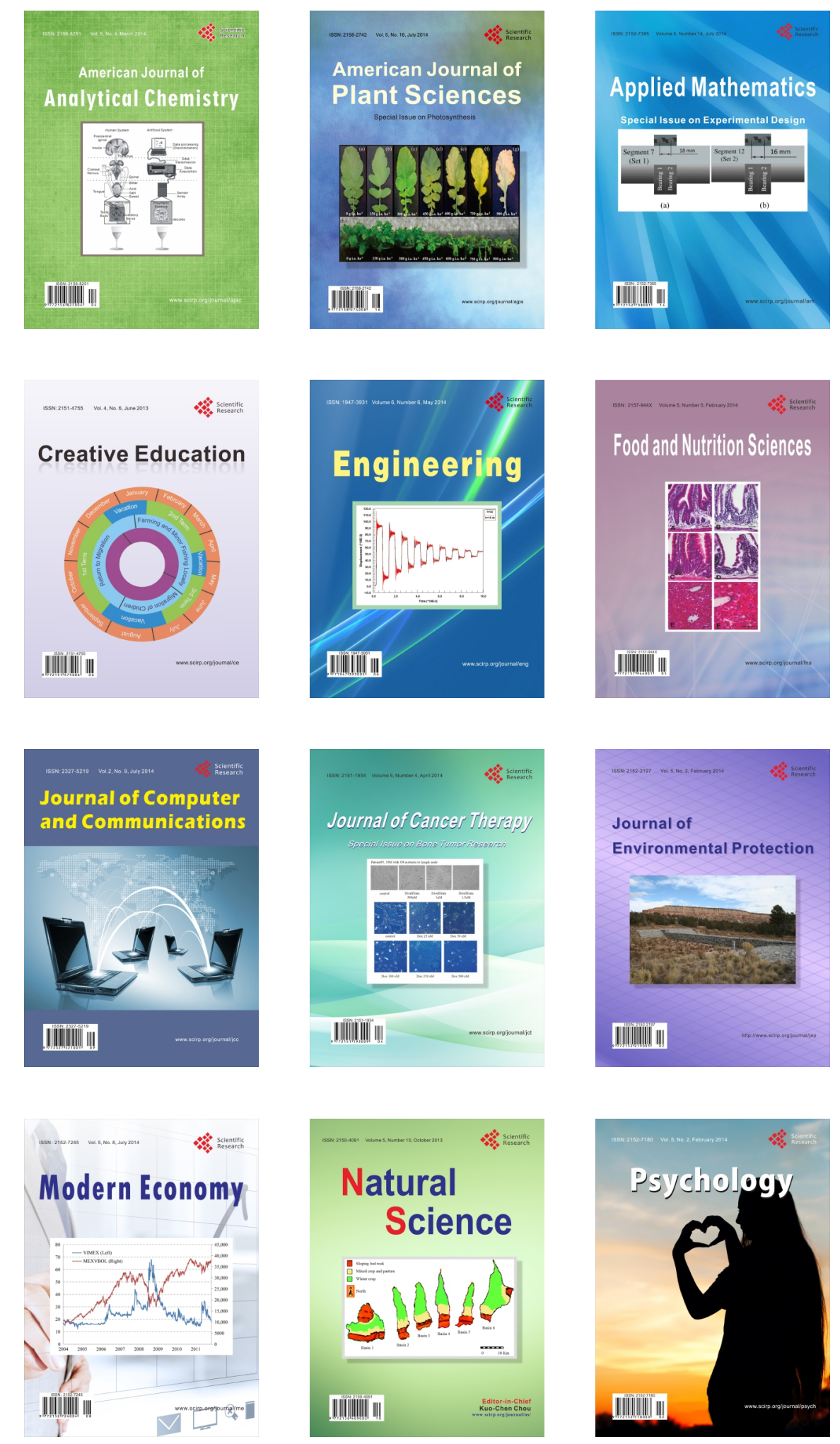